\title{
STATUS QUO DA ENGENHARIA CLÍNICA: DESAFIOS, METAS E EXPECTATIVAS ${ }^{1}$
}

\author{
STATUS QUO OF CLINICAL ENGINEERING: \\ CHALLENGES, GOALS AND EXPECTATIONS
}

\author{
João Alexandre Bisognin Felice ${ }^{2}$ e Luiz Fernando Rodrigues Junior ${ }^{3}$
}

\section{RESUMO}

Novos equipamentos médicos são criados ou atualizados com uma frequência nunca antes vista. Como forma de garantir que estas tecnologias funcionem e sejam aplicadas de forma segura dentro de hospitais e clínicas, há cerca de 50 anos foi criada a profissão de engenheiro clínico. No Brasil, a engenharia clínica existe há pouco mais de 20 anos, e tem crescido muito, porém ainda enfrenta dificuldades em sua expansão. Neste trabalho foi desenvolvido um questionário, que foi enviado por meio eletrônico à profissionais atuantes na área, a fim de obter dados a respeito do dia-a-dia da engenharia clínica, suas dificuldades e expectativas, com o intuito de alcançar o status quo da engenharia clínica no Brasil e proporcionar uma visão atualizada sobre este setor, tanto para acadêmicos quanto hospitais, com ou sem um setor de engenharia. A aplicação do questionário se mostrou efetiva, e foram obtidas respostas de todo o país, com dados como tempo de atuação do setor, equipamentos gerenciados, cronograma de manutenções preventivas e corretivas, conhecimento de normas, além das principais dificuldades. Gerando assim, um relatório que demostra um panorama atualizado da engenharia clínica no Brasil, através do qual é possível observar e concluir sobre as principais características e problemas enfrentados pela engenharia clínica no Brasil.

Palavras-chave: Gerenciamento, Hospitais, Manutenção, Panorama, Questionário.

\section{ABSTRACT}

New medical devices are created or updated at such a frequency never seen before. To ensure that these technologies work and are safely applied within hospitals and clinics, around 50 years ago, the clinical engineer was created. In Brazil, clinical engineering has existed for more than 20 years, and has grown a lot, but still faces some difficulties in expanding. In this work, a questionnaire was developed and sent to professionals in the field of clinical engineering to obtain data about its daily routine, difficulties and expectations, in order to acquire the status quo of clinical engineering in Brazil and provide an updated view on this sector, both to academics and hospitals, with or without an engineering sector. The application of the questionnaire was effective, and responses were obtained from all over the country, with data about the time since the creation of the engineering department, equipment managed, preventive and corrective maintenance chronograms, knowledge of standards, as well as the main difficulties. Thus, generating a report that shows an updated panorama on clinical engineering in Brazil, through which it is possible to understand about Brazil's clinical engineering main characteristics and difficulties.

Keywords: Hospitals, Maintenance, Management, Panorama, Questionnaire.

\footnotetext{
${ }^{1}$ Trabalho Final de Graduação II.

${ }^{2}$ Acadêmico do Curso de Engenharia Biomédica - Centro Universitário Franciscano. E-mail: joao.felice@hotmail.com.

${ }^{3}$ Orientador - Centro Universitário Franciscano. E-mail: luiz.fernando@unifra.br
} 


\section{INTRODUÇÃO}

Equipamentos médicos são cada vez mais importantes para fornecer e melhorar a saúde da população. Nos últimos 70 anos, a tecnologia moderna tem evoluído e criado novos dispositivos com uma frequência nunca antes vista, tornando técnicas médicas, antes tidas como padrão, em obsoletas (WHO, 2010).

Com esse aumento em número e complexidade dos equipamentos médicos nos hospitais, na década de 1960, nos Estados Unidos, os acidentes decorrentes da falta de conhecimento sobre a operação e manutenção destes equipamentos se tornaram corriqueiros em hospitais. Nesse contexto, engenheiros passaram a atuar nos hospitais e clínicas no gerenciamento de equipamentos e no treinamento das equipes, reduzindo acidentes e tempo de equipamento parado (BRONZINO, 2004). Já na década de 1970, com a expansão dos setores de engenharia em hospitais, esses profissionais ganharam a denominação de Engenheiros Clínicos (BRONZINO, 2004), e no início da década de 1990 foi criada a Sociedade Americana de Engenharia Clínica (American College of Clinical Engineering, ACCE), que, em 1992, definiu o engenheiro clínico como o profissional que, através da aplicação de técnicas de engenharia e gestão, apoia e melhora o cuidado do paciente (ACCE, 1992).

No Brasil, a engenharia clínica começou no final da década de 1980 e início de 1990, por iniciativa do governo federal, que decidiu na criação após o resultado de uma pesquisa que constatou que aproximadamente $1 / 3$ dos equipamentos médicos do país estavam parados por falta de manutenção (WANG; CALIL, 1991). Nesta época, a manutenção dos equipamentos, se realizada, ficava a cargo das fabricantes, cujos representantes nem sempre tinham a intenção de resolver o problema (RAMÍREZ; CALIL, 2000).

Na década de 1990, cursos de especialização em engenharia começaram a ser criados, primeiramente nos estados de São Paulo, Paraíba e Rio Grande de Sul, posteriormente se espalhando pelo país. Em 1994, a ACCE passou a permitir a certificação de engenheiros clínicos no Brasil, criando assim um indicador de qualidade para o setor no Brasil (RAMÍREZ; CALIL, 2000). Juntamente com a acreditação da ACCE, o Ministério da Saúde passou a aprovar normas que regulamentavam a produção, comercialização e manutenção de equipamentos médicos no Brasil, como a norma NBR IEC 601-1 de 1994, baseada na norma internacional de mesmo número, que regulamentava a segurança de equipamentos eletromédicos (RAMÍREZ; CALIL, 2000).

Mesmo após incentivo governamental na década de 1990 e os grandes avanços na área de engenharia clinica durante os anos 2000, segundo Araki (2017) quase 30 anos após a inserção da engenharia clínica no Brasil, apenas 10\% dos hospitais do país possuem um setor de engenharia e manutenção estruturado e atuante na manutenção e gerenciamento de equipamentos, na gestão de riscos e na redução de custos operacionais. Conforme demonstrado por SOUZA (2012), a instalação de um setor de engenharia clinica pode reduzir drasticamente os custos operacionais e de manutenção de um hospital, que conforme demonstrado em seu artigo, a economia anual gerada pelo setor de engenharia clínica pode chegar a 40\%. 
Logo após a chegada da engenharia clínica ao Brasil, agências governamentais, com o intuito de regularizar, padronizar e auxiliar na atuação da engenharia clínica, dos fabricantes e demais profissionais que trabalham com equipamentos médicos, órgãos governamentais e internacionais criaram normas e regulamentações. Nos últimos anos, desde a criação da Agencia Nacional de Vigilância Sanitária (ANVISA) em 1999, com o objetivo de fiscalizar e regulamentar, entre outras áreas, os equipamentos, locais e equipes atuantes nos hospitais e clínicas do país (BRITO, 2004), criou Resoluções de Diretoria Colegiada (RDC), abrangendo diversas áreas da saúde, como nas RDCs 07/2010, 063/2011 e 015/2012 que, juntamente com outras normas da Associação Brasileira de Normas Técnicas (ABNT), regulam o funcionamento de áreas especificas do hospital. Outras RDCs e normas da ABNT, como a RDC 050/2002 e ABNT NBR 60601:2014, que regulamentam a construção, instalação e manutenção de equipamentos médicos, a fim de garantir a máxima eficiência e qualidade no atendimento ao paciente, além de garantir a segurança de todos os profissionais envolvidos.

Objetivando obter dados sobre a engenharia clínica no Brasil, como sua abrangência, importância dentro da instituição e adequação aos padrões atuais, este trabalho busca diretamente com profissionais atuantes na área, informações de forma a gerar um relatório que demonstre o status quo da engenharia clínica no país. E desta forma proporcionar a profissionais e acadêmicos da área uma visão atualizada da engenharia clínica, as dificuldades enfrentadas e as expectativas para o futuro.

\section{MATERIAIS E MÉTODOS}

Para a obtenção dos resultados sobre o status quo da engenharia clínica, foi desenvolvido um questionário com 50 perguntas, baseado nos modelos de pesquisa por questionário propostos por Nogueira (2002), Calil (1998) e Levin-Epstein (2009). O questionário desenvolvido foi dividido em 6 partes, descritas conforme a tabela 1, e aplicado a profissionais atuantes na área e aos responsáveis pelos setores de engenharia clínica dos hospitais.

Tabela 1 - Divisão do questionário aplicado.

\begin{tabular}{ll}
\hline Dados Pessoais & $\begin{array}{l}\text { Tem por objetivo a obtenção de dados a respeito da formação e atuação do profissional, } \\
\text { além de dados para contato. }\end{array}$ \\
\hline Hospital & $\begin{array}{l}\text { Obtenção de dados a respeito do hospital, como tamanho, número de leitos, localização e } \\
\text { detalhes sobre a atuação do profissional na área. }\end{array}$ \\
\hline Setor de Engenharia Clínica & $\begin{array}{l}\text { Esta seção visa obter dados sobre o setor como: tempo de atuação, número de profissionais } \\
\text { no setor e espaço destinado. }\end{array}$ \\
\hline Equipamentos e Manutenção & $\begin{array}{l}\text { Visa obter dados sobre a gestão dos equipamentos, rotinas de manutenção, normas e ferra- } \\
\text { mentas utilizadas diariamente, treinamentos realizados, investimentos e metodologias. }\end{array}$ \\
\hline Dificuldades & $\begin{array}{l}\text { Informaçães sobre como o setor é visto dentro do hospital e suas principais dificuldades } \\
\text { quanto a funcionários, infraestrutura e financiamento. }\end{array}$ \\
\hline Expectativas & Expectativas e visão do futuro da engenharia clínica e do hospital \\
\hline
\end{tabular}


O questionário foi aplicado em ambiente virtual, por meio da ferramenta de avaliação Google Forms $^{\circledR}$, escolhido pela sua forma fácil e interativa para criação e resposta dos formulários, além de suas ferramentas de gerenciamento das respostas obtidas e tratamento de dados em tabelas e gráficos. O formulário foi enviado por meio eletrônico a profissionais da área e compartilhado nas mídias sociais e grupos de engenharia clínica. Após a obtenção das respostas os dados foram tratados nos softwares Google Forms ${ }^{\circledR}$ e Microsoft Excel ${ }^{\circledR}$, devido a suas extensas ferramentas para análise de dados. O questionário foi validado em duas etapas: $1^{\text {a }}$ o questionário foi apresentado a um profissional da área de publicidade e propaganda. Nesse caso a validação tinha como objetivo verificar se esse era claro e não apresentava questionamentos dúbios. Na $2^{\text {a }}$ etapa o questionário foi enviado a um engenheiro clínico que observou se as perguntas eram adequadas e se eram condizentes com a expectativa para um setor de engenharia clínica. A elaboração, validação e aplicação do questionário foi realizada conforme fluxograma da Figura 1.

Figura 1 - Fluxograma do Projeto

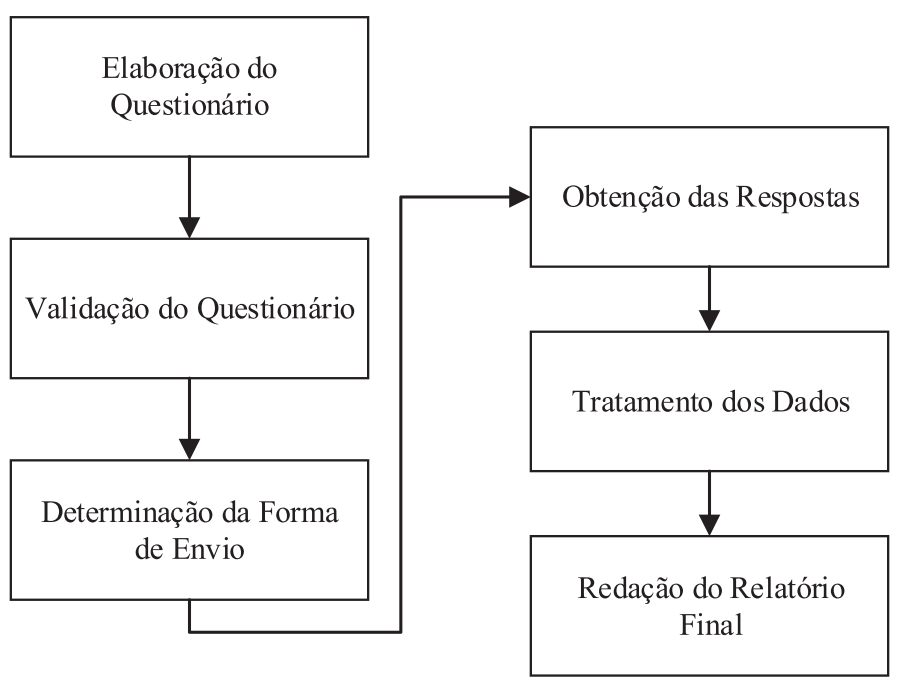

Fonte: Construção do autor.

\section{RESULTADOS E DISCUSSÕES}

Foram obtidas 32 respostas ao questionário, das quais, 31 foram utilizadas para este artigo, pois uma apresentou dados referentes a outro país. As respostas obtidas, de acordo com a região do Brasil, dispostas na Figura 2, demonstram um panorama do país.

Dos respondentes, nota-se uma grande presença de engenheiros elétricos/eletrônicos (45\%) e engenheiros biomédicos (16\%). Os outros 39\% se dividem em outras engenharias, como controle e automação $(9,7 \%)$ e tecnólogos de diversas áreas. Quanto a nível de pós-graduação, 45\% possui 
especialização em engenharia clínica e $12 \%$ possui mestrado ou doutorado. Nota-se também que $25 \%$ dos respondentes já atuam na área a mais de 15 anos, enquanto $57 \%$ possuem até 10 anos, que juntamente ao fato de aproximadamente $40 \%$ dos respondentes possuir até 30 anos, demonstra o quão recente é a engenharia clínica no Brasil. Estes resultados se mostram coerentes com os obtidos no questionário internacional aplicado por Glouhova e Pallikarakis (2004), onde os respondentes da américa latina, em sua maioria, possuem especialização em engenharia clínica e que, em média os respondentes tinham entre 23 e 38 anos e tinha no máximo 13 anos de experiência na área. Estes resultados se mostram coerentes com os obtidos no questionário internacional aplicado por Glouhova e Pallikarakis (2004), onde os setores da américa latina possuem um número de engenheiros muitas vezes maior que o de técnicos. Ainda, sobre o nível de formação, houve um aumento significativo no número de pessoas com especialização, mestrado ou doutorado, dado que no estudo original, menos de $10 \%$ dos respondentes possuíam pós-graduação. Já a idade média e o tempo de experiencia não sofreu alterações significativas entre o estudo de Glouhova e Pallikarakis e este trabalho, dadas as idades médias de 31 anos com 7 anos de experiencia na área. A similaridade entre os dois trabalhos demonstra que, mesmo após mais de 15 anos da realização do estudo, a face da engenharia clínica na américa latina, em especial do Brasil, não sofreu grandes alterações, sendo notado um aumento apenas no nível de pós-graduação.

Dos hospitais analisados, aproximadamente $52 \%$ possui menos de 7 anos desde sua criação (Figura 3) e 37,3\% possuem menos de 10 anos. Nos últimos anos a engenharia clínica tem sido criada em hospitais de todos os tamanhos, porém percebe-se que, dos hospitais analisados, que possuem mais de 20 anos de engenharia clínica, todos possuem porte grande (acima de 150 leitos) ou extra (acima de 500 leitos).

Figura 2 - Distribuição das respostas por região do Brasil.

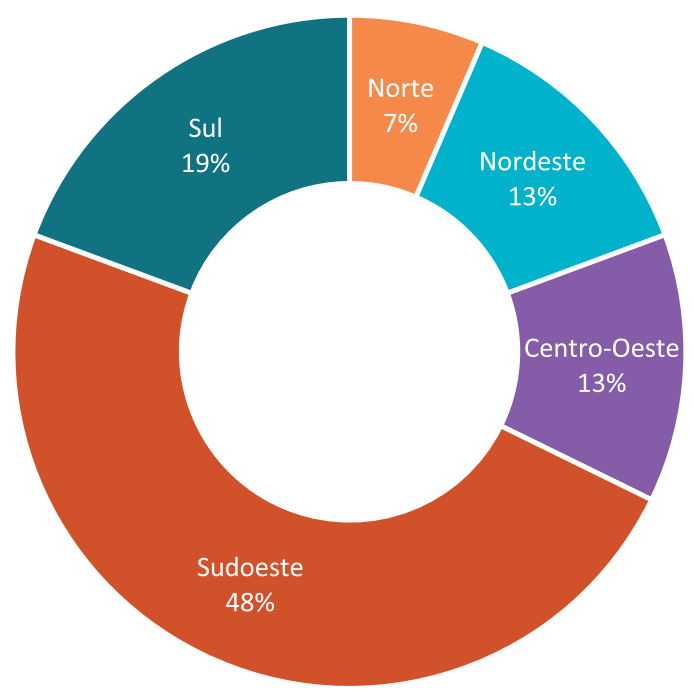

Fonte: Construção do autor. 
Figura 3 - Criação de setores de engenharia clínica por década

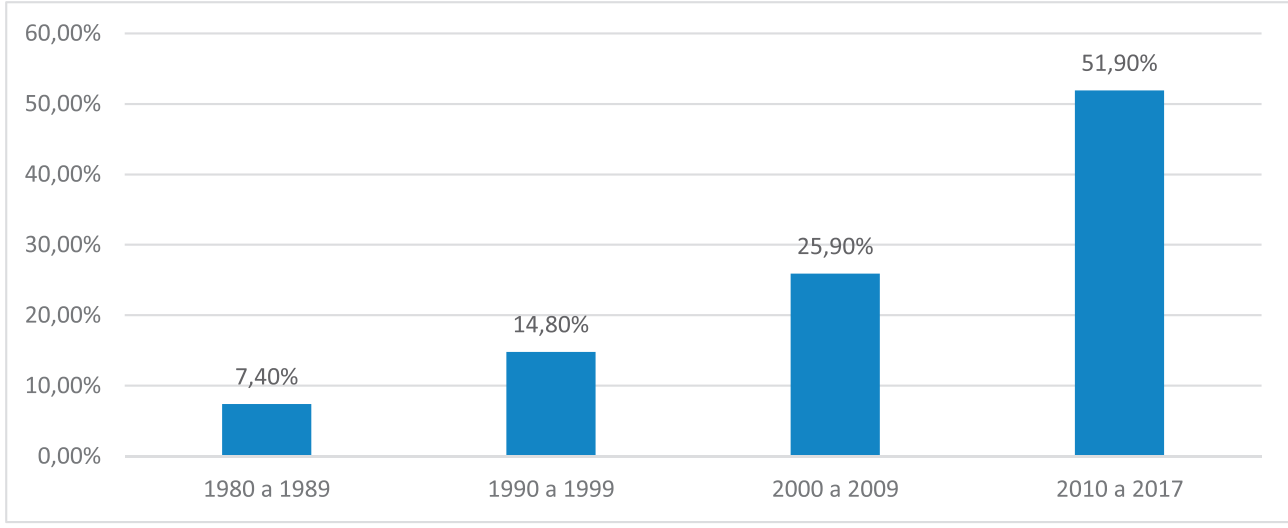

Fonte: Construção do autor.

Surpreendentemente, sobre o número de profissionais atuantes no setor, incluindo engenheiros, técnicos, estagiários e outros profissionais, não houve correlação entre o número total e o porte do hospital. Assim como do tamanho destinado ao setor, que em geral não atende à demanda dos setores, e não cresce conforme o tamanho da equipe ou carga de trabalho, tornando os espaços apertados, mesmo em hospitais de grande porte.

Dos hospitais analisados, à exceção dos de porte extra, cujo número de equipamentos sob gerencia da engenharia clínica pode facilmente passar de 4000 itens, a média de equipamentos foi de 1200, variando muito pouco dos hospitais de pequeno, médio e grande porte. Dos tipos de equipamentos gerenciados, classificados de acordo com o Ministério da Saúde (2017), nota-se pela Figura 4 que os equipamentos em maior número são os equipamentos de manutenção da vida e de diagnóstico por imagem, o que está dentro do esperado devido à necessidade e o elevado número destes equipamentos em hospitais. Um dado que chama atenção é o fato de apenas $26 \%$ dos setores serem responsáveis por equipamentos de infraestrutura, que abrange equipamentos como ar-condicionado central, usina de oxigênio e geradores, demonstra que, conforme Calil (2016), a engenharia clínica em países como o Brasil, tem um foco centrado na manutenção de equipamentos como forma de redução de custos de terceirização ou assistência do fabricante, sendo essa redução mais significativa quando aplicada a equipamentos menores, de menor complexidade e em maior número no hospital. 
Figura 4 - Tipos de equipamentos sob gerência da engenharia clínica

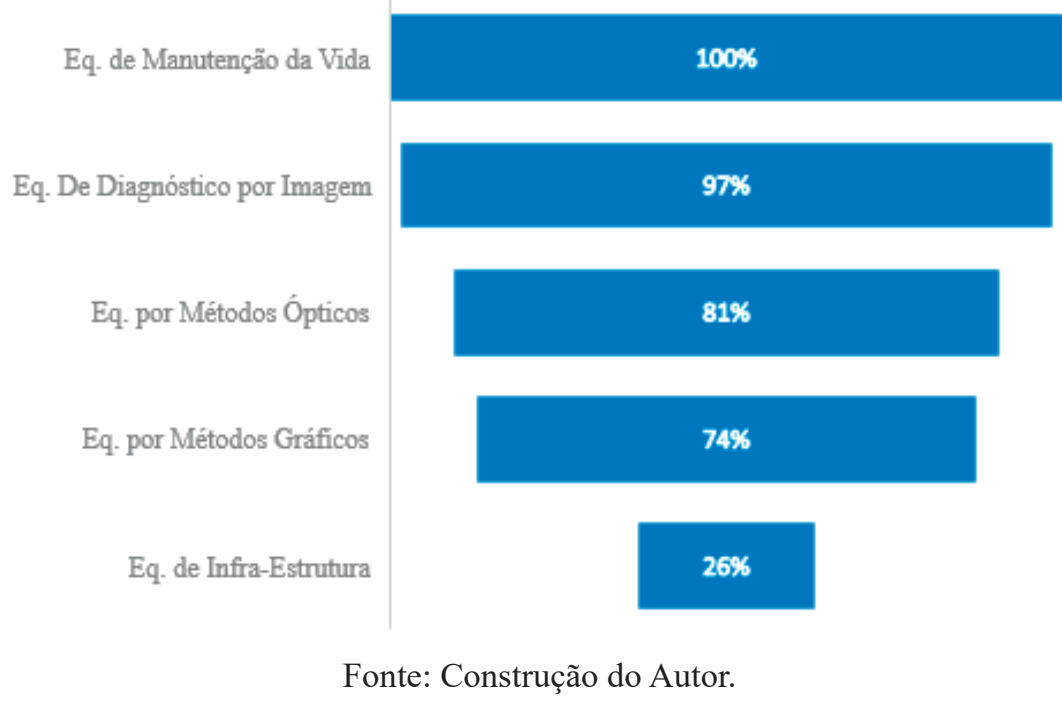

Sendo o número médio de equipamentos monitorados pelos setores aproximadamente 1200, o número de OSs (Ordens de Serviço) recebidas pelos setores respondentes pode parecer irreal, com hospitais com 2700 equipamentos monitorados, apresentando até 22000 ordens de serviço por ano (em 2016), e hospitais com 4000 equipamentos apresentando apenas 6500 OSs, um número consideravelmente menor se comparados os números de equipamentos. Em um panorama geral dos respondentes, o número médio de OSs recebidas em 2016 foi de 3600, com média de 300 ordens mensais. Esses números demonstram a importância do setor de engenharia clínica e seu grande fluxo de atividades diárias. Além disso, pode-se perceber que as equipes em saúde dos hospitais não têm hesitado em chamar a manutenção em caso de problemas na operação dos equipamentos, demonstrando a interação entre setores de saúde e de manutenção nos hospitais. Para os hospitais com números muito elevados de ordens de serviço em relação aos equipamentos gerenciados, a diferença pode ser dada pelo fato de que o sistema informatizado de alguns hospitais agrupa OSs de diversos setores, dificultando a sua contabilização, ou por motivos além do escopo deste trabalho.

Quanto as manutenções dos equipamentos, conforme apresentado pelo gráfico na Figura 5, os equipamentos com maior índice de manutenções corretivas seguem o apresentado na figura anterior, sendo proporcionais ao número de equipamentos do tipo no hospital. 
Figura 5 - Manutenções corretivas por tipo de equipamento

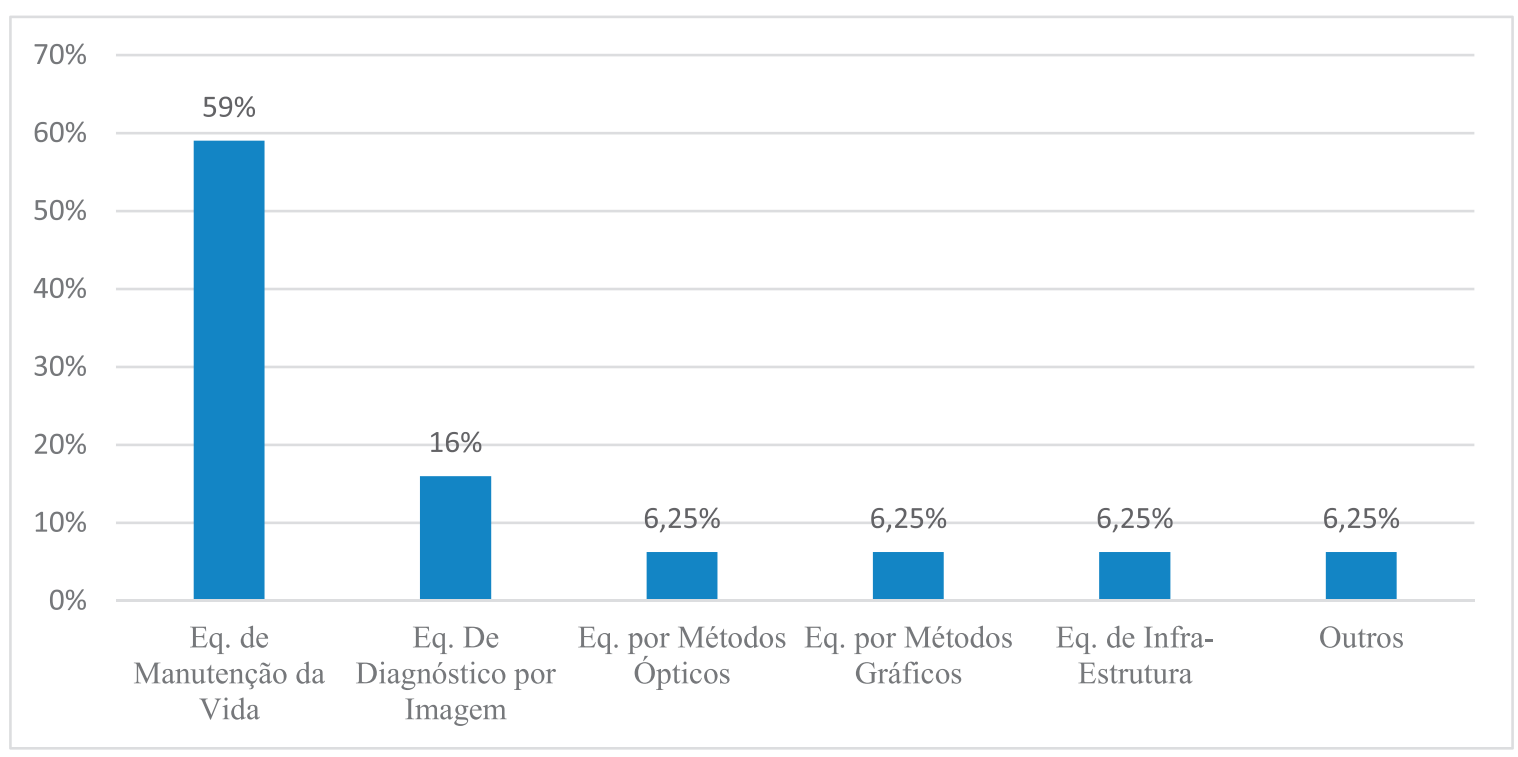

Fonte: Construção do autor.

Das manutenções destes equipamentos, seguindo o que foi dito por Calil (2016), onde a principal meta da engenharia clínica no Brasil é reduzir custos com manutenções corretivas e terceirizações, percebe-se com os dados da Figura 6, que apresenta a relação entre manutenções INTERNAS / EXTERNAS realizadas no hospital, sendo manutenções internas as feitas pela própria equipe de engenharia ou outro setor responsável no hospital e manutenções externas às realizadas fora do hospital e/ou por empresa terceirizada (fabricante do equipamento ou outra empresa autorizada), que os grupos de engenharia clínica têm tentado reduzir seus custos através da redução nas terceirizações de manutenções, realizando até $75 \%$ de suas manutenções internamente.

Figura 6 - Relação entre manutenções Internas / Externas realizadas no hospital.

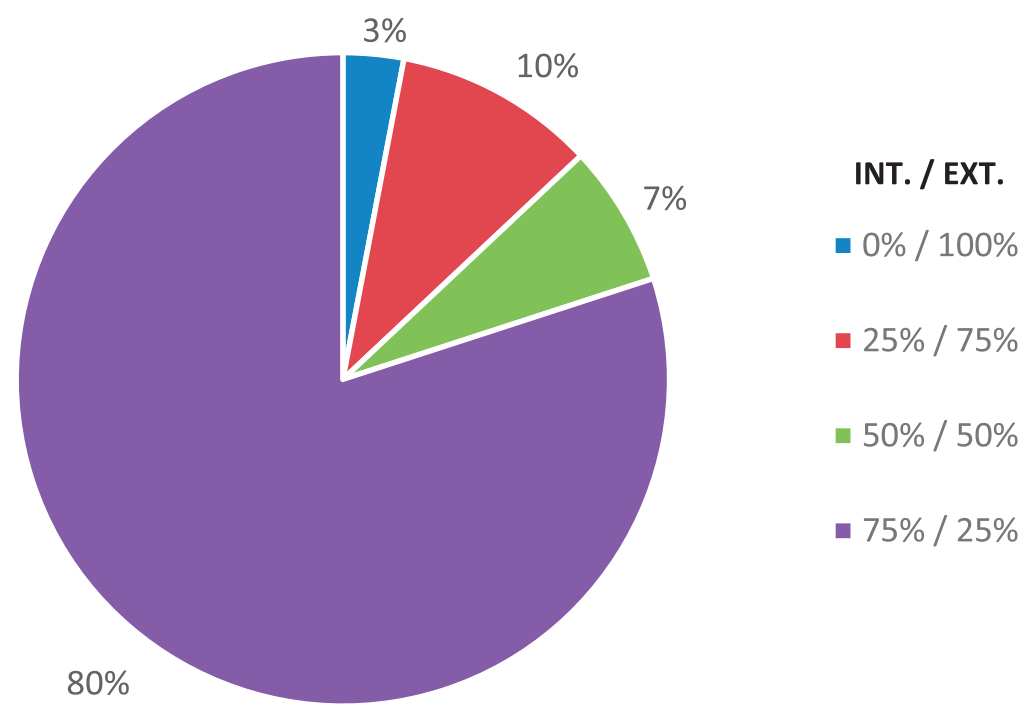

Fonte: Construção do autor. 
Outro dado interessante é o fato de que os grupos apresentaram taxas de manutenções corretivas e preventivas realizadas internamente próximas a 50\% (Figura 7), demonstrando que a manutenção preventiva tem crescido e sua importância, tanto econômica quanto para segurança e confiabilidade do equipamento, está sendo notada pelos hospitais.

Figura 7 - Relação entre manutenções corretivas e preventivas realizadas internamente.

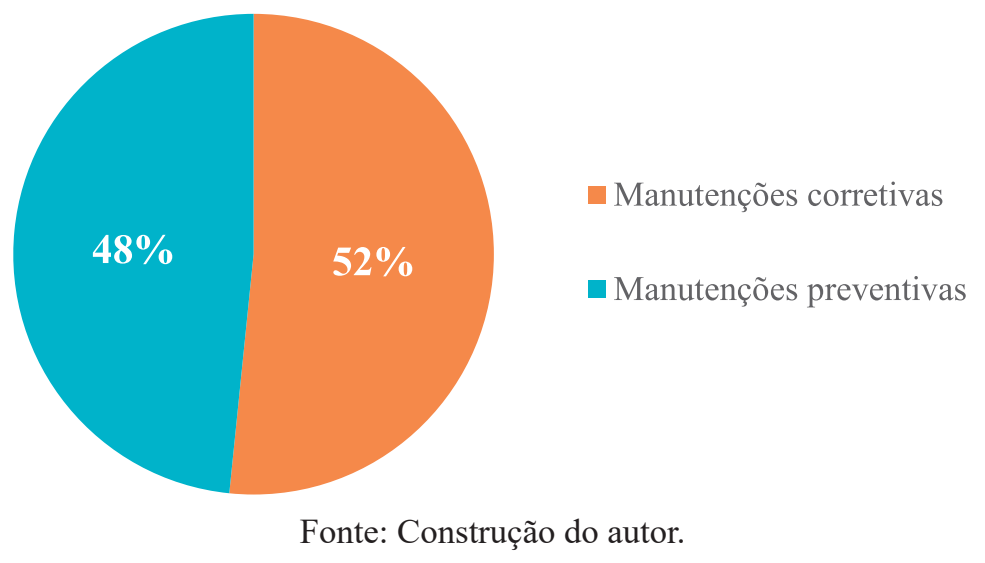

Das manutenções externas, nota-se que essas são em sua maioria preventivas e corretivas baseadas em um contrato prévio de manutenção, seja com a fabricante ou outra empresa autorizada (Figura 8), principalmente por motivos de garantia e limitações impostas pelo fabricante quanto ao pessoal autorizado a realizar manutenção em certos equipamentos, principalmente de alta complexidade. Das manutenções corretivas externas, nota-se sua redução, principalmente devido as reduções de custos (GOMES TERRA et al., 2014), porém a redução total destas é dificultada principalmente pela quantidade de equipamentos obsoletos presentes nos hospitais, cuja manutenção está reduzida a apenas algumas empresas especializadas.

Figura 8 - Relação entre as taxas de manutenção externa por demanda e por contrato prévio.

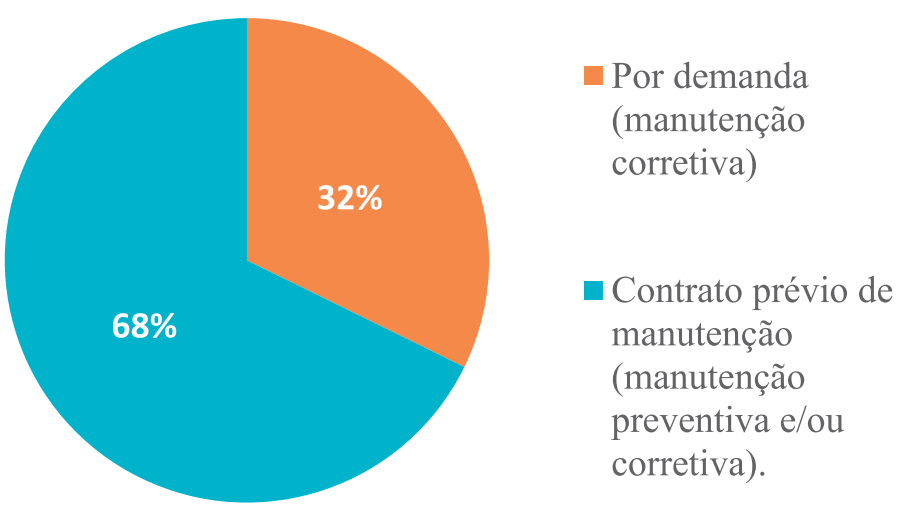

Fonte: Construção do autor.

Referente as manutenções preventivas realizadas internamente, os grupos de engenharia clínica analisados apresentaram seus principais critérios para definição da frequência das manutenções corretivas, sendo a principal delas a recomendação do fabricante. Outros critérios tomados para de- 
terminação da frequência são normas e legislações (estaduais, nacionais e internacionais), e experiência da equipe ou regulamentações internas do hospital.

Sobre a frequência das calibrações dos equipamentos, tanto de medição quanto equipamentos médicos, mostrou que aproximadamente $50 \%$ dos setores utilizam normas como principal critério para determinação da frequência.

Sendo as normas tão importantes na definição das frequências de calibração e manutenção corretiva, foi analisado se os setores estão familiarizados com algumas normas nacionais emitidas pela ABNT, ANVISA e INMETRO. Os dados apresentados na Figura 9 mostram que a engenharia clínica no Brasil está, em sua maioria, seguindo as normas estabelecidas e padronizando suas atividades a fim de garantir que os equipamentos médicos possam atender com eficiência e segurança as exigências da equipe médica. A tabela 2 apresenta as normas mencionadas no questionário, uma breve descrição e os valores atualizados para o mês de 2020.

Como forma de garantir a correta manutenção, preventiva e corretiva, dos equipamentos por parte da equipe de engenharia e manutenção do hospital, são recomendados alguns itens básicos, conforme estabelecidos por CALIL e TEIXEIRA (1998). A presença destes equipamentos é apresentada pela Figura 10, que mostra que a maioria dos setores analisados possui o mínimo de equipamentos para garantir o funcionamento e a segurança dos equipamentos médicos do hospital.

Figura 9 - Normas e legislações mais utilizadas pelos setores de engenharia clínica.

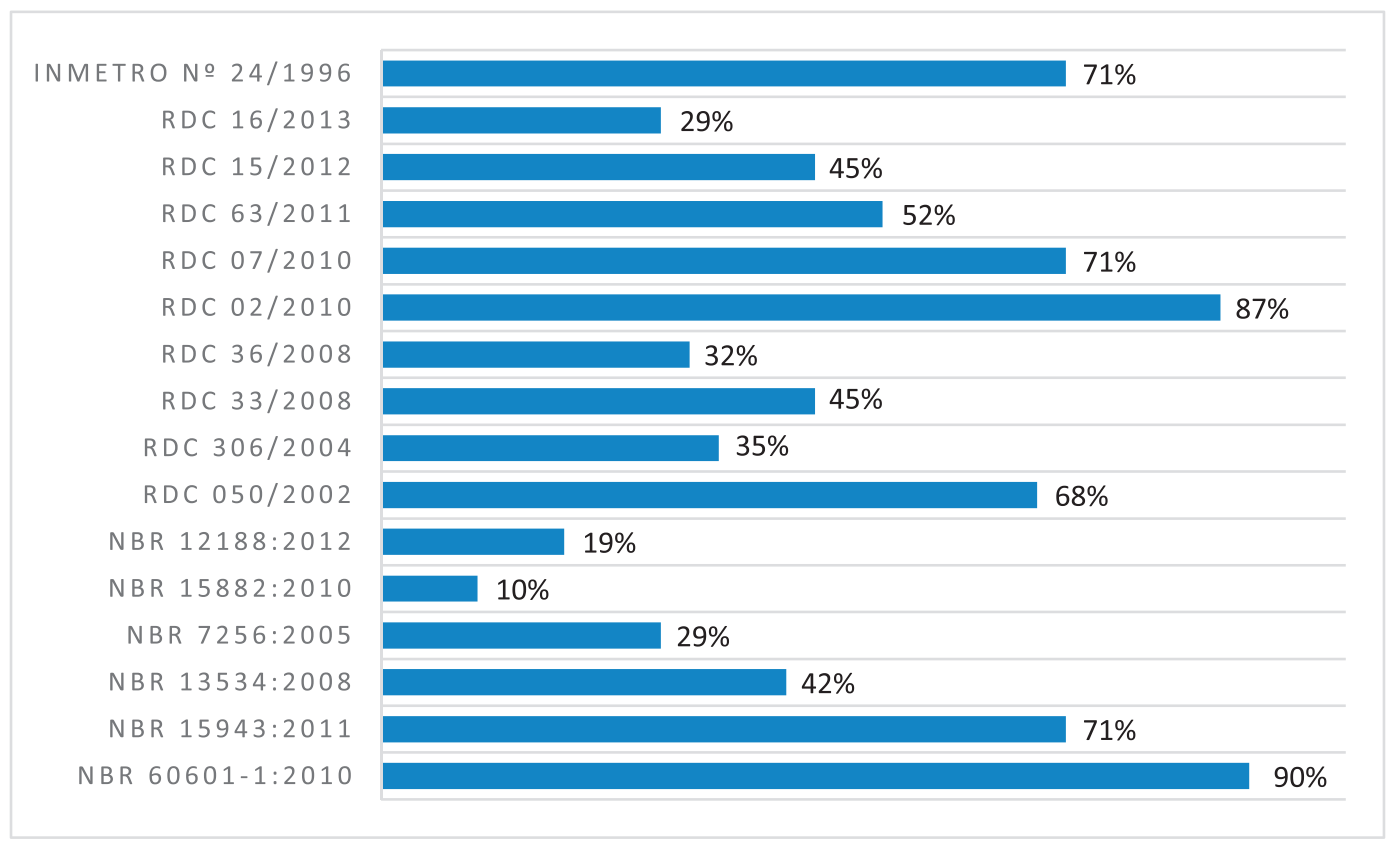

Fonte: Construção do autor. 
Tabela 2 - Normas mais utilizadas na engenharia clínica e sua respectiva descrição.

\begin{tabular}{|c|c|c|}
\hline Norma & Breve descrição & Custo (R\$)* \\
\hline $\begin{array}{l}\text { INMETRO } \\
N^{\circ} 216 / 2008\end{array}$ & Estabelece condições mínimas para calibração de esfigmomanômetros & Gratuita \\
\hline RDC 16/2013 & Boas práticas de fabricação de produtos médicos & Gratuita \\
\hline RDC $15 / 2012$ & Boas práticas para o processamento de produtos para saúde & Gratuita \\
\hline RDC 63/2011 & Boas práticas de funcionamento para os serviços da saúde & Gratuita \\
\hline RDC 07/2010 & Requisitos mínimos para o funcionamento de Unidades de Terapia Intensiva & Gratuita \\
\hline RDC $02 / 2010$ & Gerenciamento de tecnologias em saúde em estabelecimentos de saúde & Gratuita \\
\hline RDC $36 / 2008$ & Regulamento técnico para funcionamento dos serviços de atenção obstétrica e neonatal & Gratuita \\
\hline RDC $33 / 2008$ & Regulamento técnico para sistemas de tratamento e distribuição de água para hemodiálise & Gratuita \\
\hline RDC 306/2004 & Regulamento técnico para o gerenciamento de resíduos de serviços de saúde & Gratuita \\
\hline RDC 50/2002 & Regulamento técnico de projetos físicos de estabelecimentos de assistenciais de saúde & Gratuita \\
\hline $\begin{array}{l}\text { ABNT NBR } \\
12188: 2016\end{array}$ & Sistemas centralizados de suprimento de gases medicinais & $\mathrm{R} \$ 158,90$ \\
\hline $\begin{array}{l}\text { ABNT NBR } \\
15882: 2010\end{array}$ & Misturador de gases para uso medicinal & $\mathrm{R} \$ 76,00$ \\
\hline $\begin{array}{l}\text { ABNT NBR } \\
7256: 2005\end{array}$ & Tratamento de ar em estabelecimentos assistenciais de saúde & $\mathrm{R} \$ 124,40$ \\
\hline $\begin{array}{l}\text { ABNT NBR } \\
13534: 2008\end{array}$ & Instalações elétricas de baixa tensão & $\mathrm{R} \$ 124,40$ \\
\hline $\begin{array}{l}\text { ABNT NBR } \\
15943: 2011\end{array}$ & $\begin{array}{l}\text { Diretrizes para um programa de gerenciamento de equipamentos de infraestrutura de servi- } \\
\text { ços de saúde e de equipamentos para saúde }\end{array}$ & $\mathrm{R} \$ 124,40$ \\
\hline $\begin{array}{l}\text { ABNT NBR IEC } \\
60601-1: 2010\end{array}$ & $\begin{array}{l}\text { Equipamento eletromédico } \\
\text { Parte 1: Requisitos gerais para segurança básica e desempenho essencial }\end{array}$ & $\mathrm{R} \$ 0,00 * *$ \\
\hline & $\begin{array}{c}* \text { Preços avaliados no mês de novembro de } 2020 . \\
* * \text { Gratuita em decorrência da pandemia de COVID-19. } \\
\text { Fonte: Construcão do autor. }\end{array}$ & \\
\hline
\end{tabular}

Figura 10 - Equipamentos considerados mínimos para manutenção e calibração de dispositivos hospitalares.

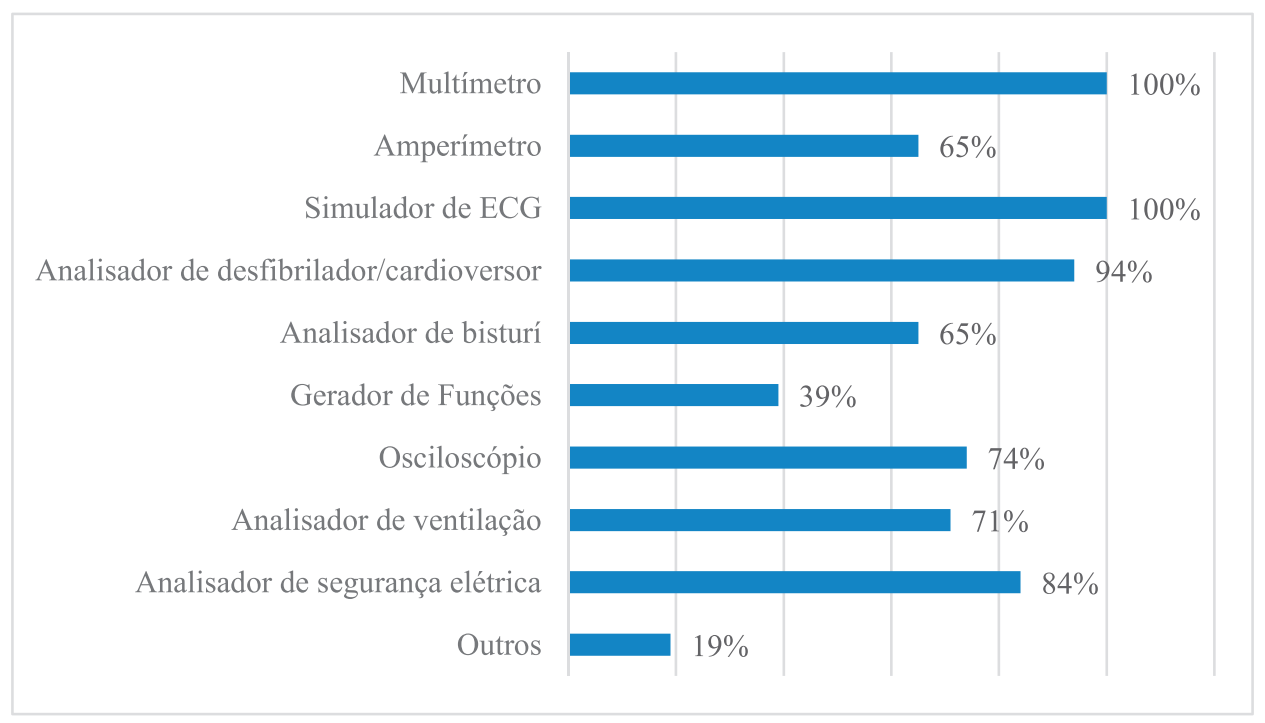

Fonte: Construção do autor.

Treinamentos de equipes são de fundamental importância para a correta utilização do equipamento e redução de problemas e acidentes. Dos hospitais respondentes, notou-se que a maioria (56\%) 
realiza treinamentos apenas na aquisição do equipamento, enquanto outros setores realizam treinamentos regulares, em muitos casos semestralmente ou anualmente. Essa baixa ou inexistente frequência no treinamento das equipes na utilização pode levar a falhas graves no equipamento, ou até acidentes.

Ainda sobre a manutenção e gerência de equipamentos médicos, foram realizadas perguntas sobre metodologias para aquisição e avaliação da obsolescência de equipamentos, onde, respectivamente, aproximadamente $75 \%$ e $70 \%$ responderam possuir algum tipo de metodologia própria para garantir que os equipamentos estejam funcionando corretamente.

Durante o questionário foram realizadas perguntas sobre as dificuldades encontradas diariamente pelas equipes de engenharia clínica (Figura 11).

Figura 11 - Principais dificuldades enfrentadas no dia a dia da engenharia clínica

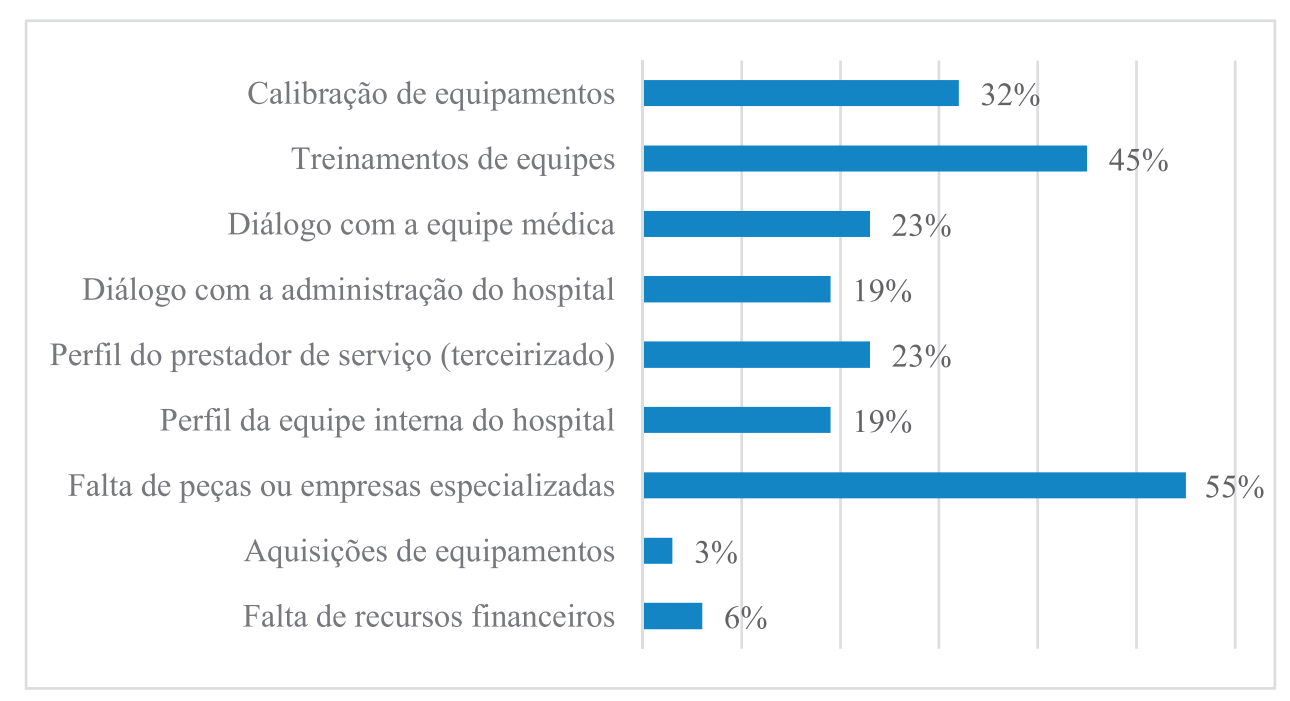

Fonte: Construção do autor.

Sobre como o setor é visto pela administração e equipe médica do hospital, todos os respondentes afirmaram serem bem vistos como uma peça fundamental para o funcionamento do hospital. Os setores também afirmam ter suas solicitações atendidas pela administração e pela equipe médica, dadas as ressalvas quanto ao orçamento limitado de alguns hospitais e limitações da equipe médica.

Como encerramento do questionário, foram feitas perguntas a respeito da visão do respondente a respeito do futuro da engenharia clínica do hospital e como área de atuação. Quanto a visão da engenharia do próprio hospital, as opiniões diferem, com algumas respostas estimando uma redução na equipe, assim como outras com expectativa de crescimento. Já a respeito da engenharia clínica como área de atuação, os respondentes se apresentaram com boas expectativas, esperando crescimento constante da área. Também foi perguntado a respeito da expectativa de crescimento do hospital, onde a maioria espera um crescimento e alguns apresentaram os projetos já em andamento para ampliação do hospital. 


\section{CONCLUSÕES}

Neste estudo foi desenvolvido um questionário com o objetivo de identificar as principais características, dificuldades e expectativas dos setores de engenharia clínica do Brasil, a fim de obter um comparativo entre os hospitais e estabelecer o status quo da engenharia clínica no Brasil.

O questionário aplicado se mostrou eficiente na obtenção dos resultados e o método de aplicação mostrou-se eficiente, com a obtenção de uma quantidade razoável de respostas. As respostas ao questionário se mostraram relevantes e foi possível estabelecer conclusões a respeito do funcionamento dos setores de engenharia clínica no Brasil, assim como suas principais dificuldades e expectativas para o futuro.

Com isso espera-se que os resultados apresentados neste trabalho possam servir para que setores de engenharia clínica espalhados pelo país que tenham ou não respondido este questionário possam tem uma base para comparação e saber se estão dentro do esperado, em relação aos hospitais do Brasil.

\section{REFERÊNCIAS}

ABNT. NBR IEC 60601:2014 - Certificação de Dispositivos Eletromédicos.

ANVISA. Resolução de Diretoria Colegiada No 050/2002 - Dispõe sobre o Regulamento Técnico para planejamento, programação, elaboração e avaliação de projetos físicos de estabelecimentos assistenciais de saúde. Brasília, 2002.

ANVISA. Resolução de Diretoria Colegiada Nº 07/2010 - Dispõe sobre os requisitos mínimos para funcionamento de Unidades de Terapia Intensiva e dá outras providências. Brasília, 2010.

ANVISA. Resolução de Diretoria Colegiada Nº63/2011 - Dispõe sobre os Requisitos de Boas Práticas de Funcionamento para os Serviços de Saúde. Brasília, 2011.

ANVISA. Resolução de Diretoria Colegiada N 015/2012 - Dispõe sobre requisitos de boas práticas para o processamento de produtos para saúde e dá outras providências. Brasília, 2012.

American College of Clinical Engineering. ACCE Definition, 1992. Disponível em: www.acce.org. Acesso em: 12/2017. 
ARAKI, F. Apenas 10\% dos Hospitais Brasileiros Possuem Departamento de Engenharia e Manutenção bem Estruturado. Revista Visão Hospitalar, p. 44-47, 2017.

BRITO, Lúcio F. de M. Clinical Engineering in Brazil. In: DYRO, J. (Ed.) Clinical Engineering Handbook. Burlington: Elsevier Academic Press, 2004. Cap. 20.

BRONZINO, J. D. Clinical Engineering: Evolution of a Discipline. In: DYRO, J. (Ed.) Clinical Engineering Handbook. Burlington: Elsevier Academic Press, 2004. Cap. 1.

CALIL, S. J.; TEIXEIRA, M. S. Gerenciamento de Manutençao de Equipamentos Hospitalares, volume 11. p. 108, 1998.

CALIL, S. J. The Evolution of Clinical Engineering: History and the Role of Technology in Health Care. In: MINIATI, R. et al. Clinical Engineering: From Devices to Systems. Elsevier Academic Press, 2016. Cap. 1.

GLOUHOVA, Mariana; PALLIKARAKIS, Nicolas. Wolrd Clinical Engineering Survey. In: DYRO, J. (Ed.) Clinical Engineering Handbook. Burlington: Elsevier Academic Press, 2004. Cap. 15.

GOMES TERRA, T. et al. Uma Revisão Dos Avanços Da Engenharia Clínica No Brasil. Disciplinarum Scientia, v. 15, n. 1, p. 47-61, 2014.

LEVIN-EPSTEIN, M. 2008 Survey of Salaries and Responsibilities for Hospital Biomedical/ Clinical Engineering and Technology Personnel. Jornal of Clinical Engineering, n. Jan./Mar., p. 42-48, 2009.

Ministério da Saúde. Data SUS, 2017. Disponivel em: https://bit.ly/3qCnXiw. Acesso em 12/2017.

NOGUEIRA, R. Elaboração e análise de questionários: uma revisão da literatura básica e a aplicação dos conceitos a um caso real. p. 1-27, 2002.

RAMÍREZ, Ernesto F. F.; CALIL, Saide J. Engenharia Clínica: Parte I - Origens (1942-1996). Semina: Ci. Exatas/Tecnol. Londrina, v. 21, n. 4, p. 27-33, dez. 2000.

SOUZA, D. B. DE; MILAGRE, S. T.; SOARES, A. B. Avaliação econômica da implantação de um serviço de Engenharia Clínica em hospital público brasileiro. Revista Braileira de Engenharia Biomédica, v. 28, p. 327-336, 2012. 
WANG, B. S.; CALIL, S. Clinical engineering in Brazil: current status. Journal of clinical engineering, 1991.

World Health Organization - WHO. MEDICAL DEVICES: Managing the mismatch: an outcome of the priority medical devices project. p. 1-36, 2010. 
